DOI:10.22337/2587-9618-2020-16-3-35-46

\title{
TRANSVERSE OSCILLATIONS OF THE BEAM ON AN ELASTIC BASE IF THE BOUNDARY CONDITIONS CHANGE
}

\author{
Yevgeny $V$. Leontiev \\ Federal autonomous institution "Main Department of State Exertise", Moscow, RUSSIA
}

\begin{abstract}
Annotation: The article deals with the proper transverse oscillations of a beam with free edges while the conditions of support on an elastic base change, taking into account its own weight and the influence of the attached mass m1. The problem of determining the forces in the beam is being solved taking into account the dynamic load $\mathrm{F}(\mathrm{t})$ applied at an arbitrary point $\mathrm{d}$ while the conditions for the support of a part of the beam on an elastic base change.

The conditions that must be taken into account while analyzing the dynamic action of the structure under the influence of variable loads in the case of changes in the conditions of support on an elastic base are formulated.
\end{abstract}

Keywords: ground base, beam on an elastic foundation, the initial parameters method, natural oscillation frequencies, forced oscillations, dynamic analysis.

\section{ПОПЕРЕЧНЫЕ КОЛЕБАНИЯ БАЛКИ НА УПРУГОМ ОСНОВАНИИ при изменении условий опирания}

\author{
Е.В.Леонтьев \\ ФАУ «Главгосэкспертиза России», г. Москва, РОССИЯ
}

\begin{abstract}
Аннотация: В работе изучаются собственные поперечные колебания балки со свободными краями при изменении условий опирания на упругое основание с учетом собственного веса и влияния присоединенной массы $\mathrm{m} 1$. Решается задача по определению усилий в балке с учетом динамической нагрузки $\mathrm{F}(\mathrm{t})$ приложенной в произвольной точке $\mathrm{d}$ при изменении условий опирания части балки на упругое основание.

Сформулированы условия, которые необходимо учитывать при анализе динамического поведения конструкции под действием переменных нагрузок в случае изменения условий опирания на упругое основание.
\end{abstract}

Ключевые слова: грунтовое основание, балка на упругом основании, метод начальных параметров, свободные колебания, вынужденные колебания, динамический анализ.

\section{INTRODUCTION}

In order to fulfill the requirements of mechanical safety of buildings and structures, which are regulated by law [1] and have been developed in modern normative and technical documents [2, 3], it is urgent to study structural systems that change the design scheme for various reasons during local destruction $[4,5,6]$. Taking into account the affecting of sudden local destruction on the stress-strain state and dynamics of structures is an urgent need for predicting their work and assessing the bearing capacity and / or stability. Such structural systems include structures lying on the ground, which can be considered in their design as beams on an elastic foundation. To date, there are a number of works $[7,8,9]$ devoted to the study of dynamic processes caused by the sudden formation of defects in beams with partial support on an elastic foundation.

\section{MODELS AND METHODS}

We consider a "beam-base" system, in which the beam was initially completely on an elastic foundation, but when a defect suddenly formed under a part of the beam, the base was excluded from power work of this structure (Figure 1). Figure 1 shows that the left side of the beam with length $\alpha L$ is located on an 
elastic foundation with a constant coefficient $r_{0}$, the right side of the beam with length $\beta L$ is cantilever. It is of interest to solve the problem of determining the natural frequencies and forms of transverse vibrations of a beam with free edges, in the case of an added mass $m_{1}$ and a dynamic load $F(t)$ applied at an arbitrary point $d$ when a part of the base under the right part of the beam suddenly has been excluded. The differential equation of forced transverse vibrations of a beam on an elastic foundation of constant cross-section, taking into account the resistance forces for any law of change of the disturbing force $q(x, t)$, has the form [9-11]:

$$
\begin{gathered}
E I \frac{\partial^{4} y(x, t)}{\partial x^{4}}+\mu \frac{\partial^{2} y(x, t)}{\partial x^{2}}+ \\
+2 \alpha \frac{\partial^{5} y(x, t)}{\partial x^{4} \partial t}+r_{0} b y(x, t)=q(x, t),
\end{gathered}
$$

where $E$ is elasticity modulus of a beam material; $I$ is inertia moment of a beam cross section, $y(x, t)$ is transverse deflection of the beam axis in the section $x ; q(x, t)$ - disturbing load that changes its value in time $t ; \mu=q / g: q$ - evenly distributed load (dead load) attached along the beam; $g$ - acceleration of gravity; $\alpha$ - coefficient characterizing internal friction of material; $r_{0} b y(x, t)$ - the intensity of the reaction of the elastic Winkler foundation that varies its values along the length of the beam $[10,11,12] ; r_{0}$ - modulus of subgrade reaction; $b$ - width of the beam.

We solved the problem in three stages using the method of initial parameters.

At the first stage, we determined the natural transverse vibrations of the beam taking into account its own weight, and at the second stage - taking into account its own weight and the added mass $m_{1}$. At the third stage, we solved the problem taking into account the disturbing force, which varies in time according to the harmonic law $F(t)=F \sin y t$ and is applied at an arbitrary point $d$. Here: $\mathrm{F}$ is the amplitude value of the disturbing force; $\gamma$ is the angular frequency of change in the disturbing force.

\section{The first stage.}

Let us determine the circular frequencies and forms of natural transverse vibrations of a beam with free edges of length $L$ and flexural rigidity $E I$ (Figure 1).

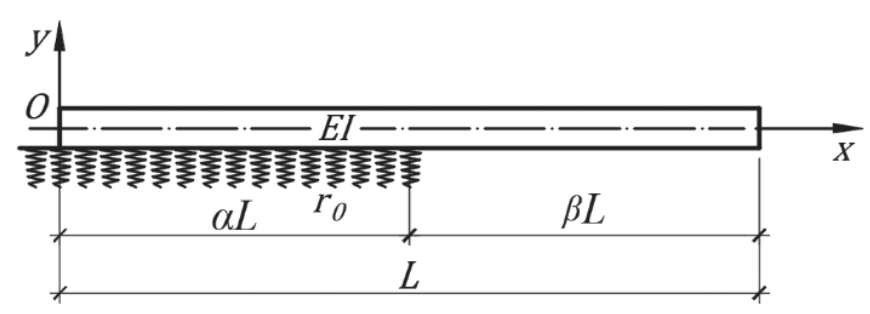

Figure 1. Beam with free edges, the left part of which $\alpha L$ is located on an elastic foundation.

It is known [13] that the dissipation of vibration energy on the frequencies and modes of natural vibrations of building structures affects only slightly, attenuation in their calculations is usually neglected.

A simple periodic solution to the equation of natural vibrations of the beam (1) is the main vibration, which changes according to the harmonic law:

$$
y(x, t)=\varphi(x) \sin (\omega t+\alpha),
$$

where $\varphi(x)$ - function that establishes the distribution law of the maximum deviations of the points of the beam axis from the equilibrium position; $\alpha$-initial phase of oscillation; $\omega=\omega_{\varkappa}$ - the circular frequency of natural transverse vibrations of the beam at the base, and $\omega=\omega_{k}$ - circular frequency of natural transverse vibrations of a beam without a base, $(\mathrm{rad} / \mathrm{s})$.

Using the method of separation of variables, problem (2) can be reduced to the equation of natural vibrations for the left side of the beam $\alpha L$ on the basis of:

$$
\varphi^{I V}(x)+\varkappa^{4} \varphi(x)=0,
$$

where we accepted designation:

$$
\varkappa^{4}=\frac{\mu \omega_{\varkappa}{ }^{2}-r}{E I} .
$$

For the right side of the beam $\beta L$ without a base, the equation of natural vibrations is:

$$
\varphi^{I V}(x)+k^{4} \varphi(x)=0,
$$

where we accepted designation:

$$
k^{4}=\frac{\mu \omega_{k}^{2}}{E I} \text {. }
$$

The solution of equations (3) and (5) is conveniently represented in the form of Krylov functions:

$$
\left.\begin{array}{l}
S(x)=\frac{1}{2}(\operatorname{ch} \lambda x+\cos \lambda x), \\
T(x)=\frac{1}{2}(\operatorname{sh} \lambda x+\sin \lambda x), \\
U(x)=\frac{1}{2}(\operatorname{ch} \lambda x-\cos \lambda x), \\
V(x)=\frac{1}{2}(\operatorname{sh} \lambda x-\sin \lambda x)
\end{array}\right\},
$$


where $\lambda=\varkappa$ corresponds to the beam laying on an elastic foundation and $\lambda=k$ corresponds to the beam without foundation.

Let us write down the values of the boundary conditions for a beam with free edges on an elastic foundation:

$$
\left.\begin{array}{l}
x=0: M(0)=Q(0)=0 \\
x=L: M(L)=Q(L)=0
\end{array}\right\}
$$

For an arbitrary section of the beam in the first section $0 \leq x_{1} \leq \alpha L$, which is located on an elastic foundation, displacements and forces are determined by the equations:

$$
\left.\begin{array}{l}
y_{1 i}\left(x_{1}\right)=y_{10 i} S\left(\varkappa_{i} x_{1}\right)+\theta_{10 i} \frac{1}{\varkappa_{i}} T\left(\varkappa_{i} x_{1}\right) \\
\theta_{1 i}\left(x_{1}\right)=y_{10 i} \varkappa_{i} V\left(\varkappa_{i} x_{1}\right)+\theta_{10 i} S\left(\varkappa_{i} x_{1}\right) \\
M_{1 i}\left(x_{1}\right)=-E J y_{10 i} \varkappa_{i}^{2} U\left(\varkappa_{i} x_{1}\right)-E J \theta_{10 i} \varkappa_{i} V\left(\varkappa_{i} x_{1}\right) \\
Q_{1 i}\left(x_{1}\right)=-E J y_{10 i} \varkappa_{i}^{3} T\left(\varkappa_{i} x_{1}\right)-E J \theta_{10 i} \varkappa_{i}^{2} U\left(\varkappa_{i} x_{1}\right)
\end{array}\right\}
$$

Here $i=1,2,3$, etc.

In the second section of the beam without a base $0 \leq x_{2} \leq \beta L$ displacements and forces for an arbitrary section are determined:

$$
\left.\begin{array}{l}
y_{2 i}\left(x_{2}\right)=y_{20 i} S\left(k_{i} x_{2}\right)+\frac{\theta_{20 i}}{k_{i}} T\left(k_{i} x_{2}\right)-\frac{M_{20 i}}{k_{i}^{2} E J} U\left(k_{i} x_{2}\right)-\frac{Q_{20 i}}{k_{i}^{3} E J} V\left(k_{i} x_{2}\right) \\
\theta_{2 i}\left(x_{2}\right)=y_{20 i} k_{i} V\left(k_{i} x_{2}\right)+\theta_{20 i} S\left(k_{i} x_{2}\right)-\frac{M_{20 i}}{k_{i} E J} T\left(k_{i} x_{2}\right)-\frac{Q_{20 i}}{k_{i}^{2} E J} U\left(k_{i} x_{2}\right) \\
M_{2 i}\left(x_{2}\right)=-E J y_{20 i} k_{i}^{2} U\left(k_{i} x_{2}\right)-E J \theta_{20 i} k_{i} V\left(k_{i} x_{2}\right)+M_{20 i} S\left(k_{i} x_{2}\right)+\frac{Q_{20 i}}{k_{i}} T\left(k_{i} x_{2}\right) \\
Q_{2 i}\left(x_{2}\right)=-E J y_{20 i} k_{i}^{3} T\left(k_{i} x_{2}\right)-E J \theta_{20 i} k_{i}^{2} U\left(k_{i} x_{2}\right)+M_{20 i} k_{i} \mathrm{~V}\left(k_{i} x_{2}\right)+Q_{20 i} S\left(k_{i} x_{2}\right)
\end{array}\right\}
$$

Using the conditions of conjugation of the sections $\alpha L$ and $\beta L$, we express the displacements and forces of the second section through the initial parameters of the first section:

$$
\left.\begin{array}{rl}
y_{2 i}\left(x_{2}\right)= & y_{10 i}\left[S\left(\varkappa_{i} \alpha L\right) S\left(k_{i} x_{2}\right)+V\left(\varkappa_{i} \alpha L\right) \frac{\varkappa_{i}}{k_{i}} T\left(k_{i} x_{2}\right)+U\left(\varkappa_{i} \alpha L\right) U\left(k_{i} x_{2}\right) \frac{\varkappa_{i}^{2}}{k_{i}^{2}}+T\left(\varkappa_{i} \alpha L\right) V\left(k_{i} x_{2}\right) \frac{\varkappa_{i}^{3}}{k_{i}^{3}}\right]+ \\
& +\theta_{10 i}\left[\frac{1}{\varkappa_{i}} T\left(\varkappa_{i} \alpha L\right) S\left(k_{i} x_{2}\right)+S\left(\varkappa_{i} \alpha L\right) \frac{1}{k_{i}} T\left(k_{i} x_{2}\right)+V\left(\varkappa_{i} \alpha L\right) U\left(k_{i} x_{2}\right) \frac{\varkappa_{i}}{k_{i}^{2}}+U\left(\varkappa_{i} \alpha L\right) V\left(k_{i} x_{2}\right) \frac{\varkappa_{i}^{2}}{k_{i}^{3}}\right] \\
\theta_{2 i}\left(x_{2}\right)= & y_{10 i}\left[k_{i} S\left(\varkappa_{i} \alpha L\right) V\left(k_{i} x_{2}\right)+\varkappa_{i} V\left(\varkappa_{i} \alpha L\right) S\left(k_{i} x_{2}\right)+\frac{\varkappa_{i}^{2}}{k_{i}} U\left(\varkappa_{i} \alpha L\right) T\left(k_{i} x_{2}\right)+\frac{\varkappa_{i}^{3}}{k_{i}^{2}} T\left(\varkappa_{i} \alpha L\right) U\left(k_{i} x_{2}\right)\right]+ \\
& +\theta_{10 i}\left[\frac{k_{i}}{\varkappa_{i}} T\left(\varkappa_{i} \alpha L\right) V\left(k_{i} x_{2}\right)+S\left(\varkappa_{i} \alpha L\right) S\left(k_{i} x_{2}\right)+\frac{\varkappa_{i}}{k_{i}} V\left(\varkappa_{i} \alpha L\right) T\left(k_{i} x_{2}\right)+U\left(\varkappa_{i} \alpha L\right) \frac{\varkappa_{i}^{2}}{k_{i}^{2}} U\left(k_{i} x_{2}\right)\right] \\
M_{2 i}\left(x_{2}\right)= & -E J y_{10 i}\left[k_{i}^{2} S\left(\varkappa_{i} \alpha L\right) U\left(k_{i} x_{2}\right)+\varkappa_{i} k_{i} V\left(\varkappa_{i} \alpha L\right) V\left(k_{i} x_{2}\right)+\varkappa_{i}^{2} U\left(\varkappa_{i} \alpha L\right) S\left(k_{i} x_{2}\right)+\frac{\varkappa_{i}^{3}}{k_{i}} T\left(\varkappa_{i} \alpha L\right) T\left(k_{i} x_{2}\right)\right]- \\
& -E J \theta_{10 i}\left[\frac{k_{i}^{2}}{\varkappa_{i}} T\left(\varkappa_{i} \alpha L\right) U\left(k_{i} x_{2}\right)+k_{i} S\left(\varkappa_{i} \alpha L\right) V\left(k_{i} x_{2}\right)+\varkappa_{i} V\left(\varkappa_{i} \alpha L\right) S\left(k_{i} x_{2}\right)+\frac{\varkappa_{i}^{2}}{k_{i}} U\left(\varkappa_{i} \alpha L\right) T\left(k_{i} x_{2}\right)\right] \\
Q_{2 i}\left(x_{2}\right)=- & E J y_{10 i}\left[k_{i}^{3} S\left(\varkappa_{i} \alpha L\right) T\left(k_{i} x_{2}\right)+\varkappa_{i} k_{i}^{2} V\left(\varkappa_{i} \alpha L\right) U\left(k_{i} x_{2}\right)+\varkappa_{i}^{2} k_{i} U\left(\varkappa_{i} \alpha L\right) V\left(k_{i} x_{2}\right)+\varkappa_{i}^{3} T\left(\varkappa_{i} \alpha L\right) S\left(k_{i} x_{2}\right)\right]- \\
- & E J \theta_{10 i}\left[\frac{k_{i}^{3}}{\varkappa_{i}} T\left(\varkappa_{i} \alpha L\right) T\left(k_{i} x_{2}\right)+k_{i}^{2} S\left(\varkappa_{i} \alpha L\right) U\left(k_{i} x_{2}\right)+\varkappa_{i} k_{i} V\left(\varkappa_{i} \alpha L\right) V\left(k_{i} x_{2}\right)+\varkappa_{i}^{2} U\left(\varkappa_{i} \alpha L\right) S\left(k_{i} x_{2}\right)\right]
\end{array}\right\}
$$


Using the boundary conditions on the right edge (8) at $x_{2}=\beta L$, we obtain the system of equations:

$$
\left\{\begin{array}{c}
M_{2 i}(\beta L)=-E J y_{10 i}\left[k_{i}^{2} S\left(\varkappa_{i} \alpha L\right) U\left(k_{i} \beta L\right)+\varkappa_{i} k_{i} V\left(\varkappa_{i} \alpha L\right) V\left(k_{i} \beta L\right)+\varkappa_{i}^{2} U\left(\varkappa_{i} \alpha L\right) S\left(k_{i} \beta L\right)+\frac{\varkappa_{i}^{3}}{k_{i}} T\left(\varkappa_{i} \alpha L\right) T\left(k_{i} \beta L\right)\right]- \\
-E J \theta_{10 i}\left[\frac{k_{i}^{2}}{\varkappa_{i}} T\left(\varkappa_{i} \alpha L\right) U\left(k_{i} \beta L\right)+k_{i} S\left(\varkappa_{i} \alpha L\right) V\left(k_{i} \beta L\right)+\varkappa_{i} V\left(\varkappa_{i} \alpha L\right) S\left(k_{i} \beta L\right)+\frac{\varkappa_{i}^{2}}{k_{i}} U\left(\varkappa_{i} \alpha L\right) T\left(k_{i} \beta L\right)\right]=0 \\
Q_{2 i}(\beta L)=-E J y_{10 i}\left[k_{i}^{3} S\left(\varkappa_{i} \alpha L\right) T\left(k_{i} \beta L\right)+\varkappa_{i} k_{i}^{2} V\left(\varkappa_{i} \alpha L\right) U\left(k_{i} \beta L\right)+\varkappa_{i}^{2} k_{i} U\left(\varkappa_{i} \alpha L\right) V\left(k_{i} \beta L\right)+\varkappa_{i}^{3} T\left(\varkappa_{i} \alpha L\right) \mathrm{S}\left(k_{i} \beta L\right)\right]- \\
-E J \theta_{10 i}\left[\frac{k_{i}^{3}}{\varkappa_{i}} T\left(\varkappa_{i} \alpha L\right) T\left(k_{i} \beta L\right)+k_{i}^{2} S\left(\varkappa_{i} \alpha L\right) U\left(k_{i} \beta L\right)+\varkappa_{i} k_{i} V\left(\varkappa_{i} \alpha L\right) \mathrm{V}\left(k_{i} \beta L\right)+\varkappa_{i}^{2} U\left(\varkappa_{i} \alpha L\right) \mathrm{S}\left(k_{i} \beta L\right)\right]=0
\end{array}\right.
$$

For a nontrivial solution of equations (12), it is necessary that the determinant, composed of the coefficients at arbitrary constants $E J y_{10}$ and $E J \theta_{10 i}$, be equal to zero:

$$
\begin{aligned}
& D=\left[k_{i}^{2} S\left(\varkappa_{i} \alpha L\right) U\left(k_{i} \beta L\right)+\varkappa_{i} k_{i} V\left(\varkappa_{i} \alpha L\right) V\left(k_{i} \beta L\right)+\varkappa_{i}^{2} U\left(\varkappa_{i} \alpha L\right) S\left(k_{i} \beta L\right)+\frac{\varkappa_{i}^{3}}{k_{i}} T\left(\varkappa_{i} \alpha L\right) T\left(k_{i} \beta L\right)\right] * \\
& *\left[\frac{k_{i}^{3}}{\varkappa_{i}} T\left(\varkappa_{i} \alpha L\right) T\left(k_{i} \beta L\right)+k_{i}^{2} S\left(\varkappa_{i} \alpha L\right) U\left(k_{i} \beta L\right)+\varkappa_{i} k_{i} V\left(\varkappa_{i} \alpha L\right) V\left(k_{i} \beta L\right)+\varkappa_{i}^{2} U\left(\varkappa_{i} \alpha L\right) S\left(k_{i} \beta L\right)\right]- \\
& -\left[\frac{k_{i}^{2}}{\varkappa_{i}} T\left(\varkappa_{i} \alpha L\right) U\left(k_{i} \beta L\right)+k_{i} S\left(\varkappa_{i} \alpha L\right) V\left(k_{i} \beta L\right)+\varkappa_{i} V\left(\varkappa_{i} \alpha L\right) S\left(k_{i} \beta L\right)+\frac{\varkappa_{i}^{2}}{k_{i}} U\left(\varkappa_{i} \alpha L\right) T\left(k_{i} \beta L\right)\right] * \\
& *\left[k_{i}^{3} S\left(\varkappa_{i} \alpha L\right) T\left(k_{i} \beta L\right)+\varkappa_{i} k_{i}^{2} V\left(\varkappa_{i} \alpha L\right) U\left(k_{i} \beta L\right)+\varkappa_{i}^{2} k_{i} U\left(\varkappa_{i} \alpha L\right) V\left(k_{i} \beta L\right)+\varkappa_{i}^{3} T\left(\varkappa_{i} \alpha L\right) S\left(k_{i} \beta L\right)\right]=0
\end{aligned}
$$

The roots of equation (13) are the countless row of values $k_{i}$ and $\varkappa$. In order to solve the equation, we introduce the relation $k_{i}=\varepsilon_{i} \varkappa_{i}$. Here $\varepsilon$ is constant value. For each root value $k_{i}$ and $\varkappa$ a certain angular frequency of natural transverse vibrations corresponds.

Using expression (4), we obtain a formula for determining $\omega_{i \varkappa}$ circular frequencies of natural transverse vibrations of a part of a beam $\alpha L$ on an elastic foundation:

$$
\omega_{i \varkappa}=\sqrt{\frac{E I \lambda_{i \mathcal{X}}^{4}}{\mu(\alpha L)^{4}}+\frac{r}{\mu}},
$$

where $\lambda_{i \varkappa}=\varkappa_{i} \alpha L$, and $i=1,2,3$ etc. - frequency sequence number.

For a part of the beam $\beta L$ without a base, using (6), we get:

where $\lambda_{i k}=k_{i} \beta L$.

$$
\omega_{i k}=\sqrt{\frac{E I \lambda_{i k}^{4}}{\mu(\beta L)^{4}}},
$$

Let us determine the natural angular frequencies of transverse vibrations of the beam parts $\alpha L$ on the base and $\beta L$ without the base, which form the spectra $\omega_{I \varkappa}$ $<\omega_{2 \varkappa}<\ldots<\omega_{n \varkappa}$ and $\omega_{1 k}<\omega_{2 k}<\ldots<\omega_{n k}$.

To determine the modes of natural vibrations, we substitute the values of the roots value $k_{i}$ and $\varkappa_{i}$ into the solution of the first equation (11), which will determine the values of the relative ordinates $i$-th of that form of natural vibrations.

\section{The second stage.}

Let us determine the natural angular frequencies and forms of transverse vibrations, taking into account the own weight and the added mass $m_{1}$ at point $d$ (Figure 2).

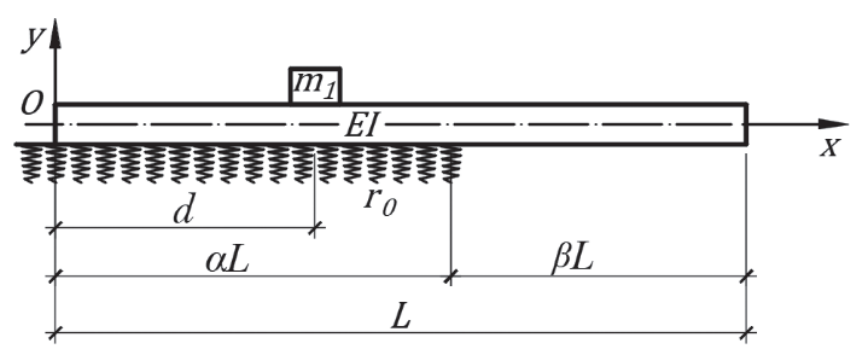

Figure 2. Beam with added mass $m_{1}$.

For an arbitrary section of the beam in the first section $0 \leq x_{1} \leq \alpha L$ displacements and forces are determined by equations (9) only up to the point of application of the mass. For $x_{1}>d$ free vibrations of the beam occur with the inertial force $I$. At point $d$ we add the inertial force $I$ and compose the system of equations: 


$$
\left.\begin{array}{l}
y_{1 i}\left(x_{1}\right)=y_{10 i} S\left(\varkappa_{i} x_{1}\right)+\frac{\theta_{10 i}}{\varkappa_{i}} T\left(\varkappa_{i} x_{1}\right)+\frac{I}{\varkappa_{i}^{3} E I} V\left(\varkappa_{i}\left(x_{1}-d\right)\right) \\
\theta_{1 i}\left(x_{1}\right)=y_{10 i} \varkappa_{i} V\left(\varkappa_{i} x_{1}\right)+\theta_{10 i} S\left(\varkappa_{i} x_{1}\right)+\frac{I}{\varkappa_{i}^{2} E I} U\left(\varkappa_{i}\left(x_{1}-d\right)\right) \\
M_{1 i}\left(x_{1}\right)=-E J y_{10 i} \varkappa_{i}^{2} U\left(\varkappa_{i} x_{1}\right)-E J \theta_{10 i} \varkappa_{i} V\left(\varkappa_{i} x_{1}\right)-\frac{I}{\varkappa_{i}} T\left(\varkappa_{i}\left(x_{1}-d\right)\right) \\
Q_{1 i}\left(x_{1}\right)=-E J y_{10 i} \varkappa_{i}^{3} T\left(\varkappa_{i} x_{1}\right)-E J \theta_{10 i} \varkappa_{i}^{2} U\left(\varkappa_{i} x_{1}\right)-I S\left(\varkappa_{i}\left(x_{1}-d\right)\right)
\end{array}\right\},
$$

$$
\text { where } I=m_{1} \omega_{i}^{2}\left[y_{0} S\left(\varkappa_{i} d\right)+\frac{\theta_{0}}{\varkappa_{i}} T\left(\varkappa_{i} d\right)\right] \text {. }
$$

Further, we have composed formulas for determining the deflections, angles of rotation, moments and shear forces of the second section of the beam without a base $0 \leq x_{2} \leq \beta L$ using (9) and the conjugation conditions:

$$
\begin{aligned}
& y_{2 i}\left(x_{2}\right)=y_{10 i}\left[S\left(\varkappa_{i} \alpha L\right) S\left(k_{i} x_{2}\right)+V\left(\varkappa_{i} \alpha L\right) \frac{\varkappa_{i}}{k_{i}} T\left(k_{i} x_{2}\right)+U\left(\varkappa_{i} \alpha L\right) U\left(k_{i} x_{2}\right) \frac{\varkappa_{i}^{2}}{k_{i}^{2}}+T\left(\varkappa_{i} \alpha L\right) V\left(k_{i} x_{2}\right) \frac{\varkappa_{i}^{3}}{k_{i}^{3}}\right]+ \\
& +\theta_{10 i}\left[\frac{1}{\varkappa_{i}} T\left(\varkappa_{i} \alpha L\right) S\left(k_{i} x_{2}\right)+S\left(\varkappa_{i} \alpha L\right) \frac{1}{k_{i}} T\left(k_{i} x_{2}\right)+V\left(\varkappa_{i} \alpha L\right) U\left(k_{i} x_{2}\right) \frac{\varkappa_{i}}{k_{i}^{2}}+U\left(\varkappa_{i} \alpha L\right) V\left(k_{i} x_{2}\right) \frac{\varkappa_{i}^{2}}{k_{i}^{3}}\right]+ \\
& +\frac{I}{E I}\left[V\left(\varkappa_{i}(\alpha L-d)\right) \frac{S\left(k_{i} x_{2}\right)}{\varkappa_{i}^{3}}+U\left(\varkappa_{i}(\alpha L-d)\right) \frac{T\left(k_{i} x_{2}\right)}{\varkappa_{i}^{2} k_{i}}+T\left(\varkappa_{i}(\alpha L-d)\right) \frac{U\left(k_{i} x_{2}\right)}{\varkappa_{i} k_{i}^{2}}+S\left(\varkappa_{i}(\alpha L-d)\right) \frac{V\left(k_{i} x_{2}\right)}{k_{i}^{3}}\right] \\
& \theta_{2 i}\left(x_{2}\right)=y_{10 i}\left[k_{i} S\left(\varkappa_{i} \alpha L\right) V\left(k_{i} x_{2}\right)+\varkappa_{i} V\left(\varkappa_{i} \alpha L\right) S\left(k_{i} x_{2}\right)+\frac{\varkappa_{i}^{2}}{k_{i}} U\left(\varkappa_{i} \alpha L\right) T\left(k_{i} x_{2}\right)+\frac{\varkappa_{i}^{3}}{k_{i}^{2}} T\left(\varkappa_{i} \alpha L\right) U\left(k_{i} x_{2}\right)\right]+ \\
& +\theta_{10 i}\left[\frac{k_{i}}{\varkappa_{i}} T\left(\varkappa_{i} \alpha L\right) V\left(k_{i} x_{2}\right)+S\left(\varkappa_{i} \alpha L\right) S\left(k_{i} x_{2}\right)+\frac{\varkappa_{i}}{k_{i}} V\left(\varkappa_{i} \alpha L\right) T\left(k_{i} x_{2}\right)+U\left(\varkappa_{i} \alpha L\right) \frac{\varkappa_{i}^{2}}{k_{i}^{2}} U\left(k_{i} x_{2}\right)\right]+ \\
& +\frac{I}{E I}\left[V\left(\varkappa_{i}(\alpha L-d)\right) \frac{k_{i} V\left(k_{i} x_{2}\right)}{\varkappa_{i}^{3}}+U\left(\varkappa_{i}(\alpha L-d)\right) \frac{S\left(k_{i} x_{2}\right)}{\varkappa_{i}^{2}}+T\left(\varkappa_{i}(\alpha L-d)\right) \frac{T\left(k_{i} x_{2}\right)}{\varkappa_{i} k_{i}}+S\left(\varkappa_{i}(\alpha L-d)\right) \frac{U\left(k_{i} x_{2}\right)}{k_{i}^{2}}\right] \\
& M_{2 i}\left(x_{2}\right)=-E J y_{10 i}\left[k_{i}^{2} S\left(\varkappa_{i} \alpha L\right) U\left(k_{i} x_{2}\right)+\varkappa_{i} k_{i} V\left(\varkappa_{i} \alpha L\right) V\left(k_{i} x_{2}\right)+\varkappa_{i}^{2} U\left(\varkappa_{i} \alpha L\right) S\left(k_{i} x_{2}\right)+\frac{\varkappa_{i}^{3}}{k_{i}} T\left(\varkappa_{i} \alpha L\right) T\left(k_{i} x_{2}\right)\right]- \\
& -E J \theta_{10 i}\left[\frac{k_{i}^{2}}{\varkappa_{i}} T\left(\varkappa_{i} \alpha L\right) U\left(k_{i} x_{2}\right)+k_{i} S\left(\varkappa_{i} \alpha L\right) V\left(k_{i} x_{2}\right)+\varkappa_{i} V\left(\varkappa_{i} \alpha L\right) S\left(k_{i} x_{2}\right)+\frac{\varkappa_{i}^{2}}{k_{i}} U\left(\varkappa_{i} \alpha L\right) T\left(k_{i} x_{2}\right)\right]- \\
& -\frac{I}{\varkappa_{i}^{3}}\left[k_{i}^{2} V\left(\varkappa_{i}(\alpha L-d)\right) U\left(k_{i} x_{2}\right)+\varkappa_{i} k_{i} U\left(\varkappa_{i}(\alpha L-d)\right) V\left(k_{i} x_{2}\right)+\varkappa_{i}^{2} T\left(\varkappa_{i}(\alpha L-d)\right) \mathrm{S}\left(k_{i} x_{2}\right)+\frac{\varkappa_{i}^{3}}{k_{i}} S\left(\varkappa_{i}(\alpha L-d)\right) T\left(k_{i} x_{2}\right)\right] \\
& Q_{2 i}\left(x_{2}\right)=-E J y_{10 i}\left[k_{i}^{3} S\left(\varkappa_{i} \alpha L\right) T\left(k_{i} x_{2}\right)+\varkappa_{i} k_{i}^{2} V\left(\varkappa_{i} \alpha L\right) U\left(k_{i} x_{2}\right)+\varkappa_{i}^{2} k_{i} U\left(\varkappa_{i} \alpha L\right) V\left(k_{i} x_{2}\right)+\varkappa_{i}^{3} T\left(\varkappa_{i} \alpha L\right) S\left(k_{i} x_{2}\right)\right]- \\
& -E J \theta_{10 i}\left[\frac{k_{i}^{3}}{\varkappa_{i}} T\left(\varkappa_{i} \alpha L\right) T\left(k_{i} x_{2}\right)+k_{i}^{2} S\left(\varkappa_{i} \alpha L\right) U\left(k_{i} x_{2}\right)+\varkappa_{i} k_{i} V\left(\varkappa_{i} \alpha L\right) \mathrm{V}\left(k_{i} x_{2}\right)+\varkappa_{i}^{2} U\left(\varkappa_{i} \alpha L\right) \mathrm{S}\left(k_{i} x_{2}\right)\right]- \\
& \left.-\frac{I}{\varkappa_{i}^{3}}\left[k_{i}^{3} T\left(k_{i} x_{2}\right) V\left(\varkappa_{i}(\alpha L-d)\right)+\varkappa_{i} k_{i}^{2} U\left(k_{i} x_{2}\right) U\left(\varkappa_{i}(\alpha L-d)\right)+\varkappa_{i}^{2} k_{i} \mathrm{~V}\left(k_{i} x_{2}\right) T\left(\varkappa_{i}(\alpha L-d)\right)+\varkappa_{i}^{3} \mathrm{~S}\left(k_{i} x_{2}\right) S\left(\varkappa_{i}(\alpha L-d)\right)\right]\right)
\end{aligned}
$$

We denote:

$$
\begin{aligned}
a_{1}= & {\left[k_{i}^{2} S\left(\varkappa_{i} \alpha L\right) U\left(k_{i} \beta L\right)+\varkappa_{i} k_{i} V\left(\varkappa_{i} \alpha L\right) V\left(k_{i} \beta L\right)+\varkappa_{i}^{2} U\left(\varkappa_{i} \alpha L\right) S\left(k_{i} \beta L\right)+\frac{\varkappa_{i}^{3}}{k_{i}} T\left(\varkappa_{i} \alpha L\right) T\left(k_{i} \beta L\right)\right] ; } \\
a_{2}= & {\left[\frac{k_{i}^{2}}{\varkappa_{i}} T\left(\varkappa_{i} \alpha L\right) U\left(k_{i} \beta L\right)+k_{i} S\left(\varkappa_{i} \alpha L\right) V\left(k_{i} \beta L\right)+\varkappa_{i} V\left(\varkappa_{i} \alpha L\right) S\left(k_{i} \beta L\right)+\frac{\varkappa_{i}^{2}}{k_{i}} U\left(\varkappa_{i} \alpha L\right) T\left(k_{i} \beta L\right)\right] ; } \\
a_{3}= & {\left[k_{i}^{2} V\left(\varkappa_{i}(\alpha L-d)\right) U\left(k_{i} \beta L\right)+\varkappa_{i} k_{i} U\left(\varkappa_{i}(\alpha L-d)\right) V\left(k_{i} \beta L\right)+\varkappa_{i}^{2} T\left(\varkappa_{i}(\alpha L-d)\right) \mathrm{S}\left(k_{i} \beta L\right)+\right.} \\
& \left.+\frac{\varkappa_{i}^{3}}{k_{i}} S\left(\varkappa_{i}(\alpha L-d)\right) T\left(k_{i} \beta L\right)\right] ; \\
a_{4}=[ & {\left[k_{i}^{3} S\left(\varkappa_{i} \alpha L\right) T\left(k_{i} \beta L\right)+\varkappa_{i} k_{i}^{2} V\left(\varkappa_{i} \alpha L\right) U\left(k_{i} \beta L\right)+\varkappa_{i}^{2} k_{i} U\left(\varkappa_{i} \alpha L\right) \mathrm{V}\left(k_{i} \beta L\right)+\varkappa_{i}^{3} T\left(\varkappa_{i} \alpha L\right) \mathrm{S}\left(k_{i} \beta L\right)\right] ; } \\
a_{5}= & {\left[\frac{k_{i}^{3}}{\varkappa_{i}} T\left(\varkappa_{i} \alpha L\right) T\left(k_{i} \beta L\right)+k_{i}^{2} S\left(\varkappa_{i} \alpha L\right) U\left(k_{i} \beta L\right)+\varkappa_{i} k_{i} V\left(\varkappa_{i} \alpha L\right) \mathrm{V}\left(k_{i} \beta L\right)+\varkappa_{i}^{2} U\left(\varkappa_{i} \alpha L\right) \mathrm{S}\left(k_{i} \beta L\right)\right] ; } \\
a_{6}= & {\left[k_{i}^{3} T\left(k_{i} \beta L\right) V\left(\varkappa_{i}(\alpha L-d)\right)+\varkappa_{i} k_{i}^{2} U\left(k_{i} \beta L\right) U\left(\varkappa_{i}(\alpha L-d)\right)+\varkappa_{i}^{2} k_{i} \mathrm{~V}\left(k_{i} \beta L\right) T\left(\varkappa_{i}(\alpha L-d)\right)+\right.} \\
& \left.+\varkappa_{i}^{3} \mathrm{~S}\left(k_{i} \beta L\right) S\left(\varkappa_{i}(\alpha L-d)\right)\right] .
\end{aligned}
$$


Using the boundary conditions on the right edge (8) at $x_{2}=\beta L$, taking into account the inertial force $I(17)$, we obtain the system of equations:

$$
\left\{\begin{array}{l}
y_{10 i}\left[a_{1}+\frac{m_{1} \omega_{i}^{2}}{\varkappa_{i}^{3} E J} S\left(\varkappa_{i} d\right) a_{3}\right]+\theta_{10 i}\left[a_{2}+\frac{m_{1} \omega_{i}^{2}}{\varkappa_{i}^{4} E J} T\left(\varkappa_{i} d\right) a_{3}\right]=0 \\
y_{10 i}\left[a_{4}+\frac{m_{1} \omega_{i}^{2}}{\varkappa_{i}^{3} E J} S\left(\varkappa_{i} d\right) a_{6}\right]+\theta_{10 i}\left[a_{5}+\frac{m_{1} \omega_{i}^{2}}{\varkappa_{i}^{4} E J} T\left(\varkappa_{i} d\right) a_{6}\right]=0
\end{array}\right.
$$

The determinant of this system:

$$
\begin{aligned}
D & =\left[a_{1}+\frac{m_{1} \varkappa_{i}}{\mu}\left(1+\frac{r}{\varkappa_{i}^{4} E J}\right) S\left(\varkappa_{i} d\right) a_{3}\right]\left[a_{5}+\frac{m_{1} \varkappa_{i}}{\mu}\left(1+\frac{r}{\varkappa_{i}^{4} E J}\right) T\left(\varkappa_{i} d\right) a_{6}\right]- \\
& -\left[a_{2}+\frac{m_{1} \varkappa_{i}}{\mu}\left(1+\frac{r}{\varkappa_{i}^{4} E J}\right) T\left(\varkappa_{i} d\right) a_{3}\right]\left[a_{4}+\frac{m_{1} \varkappa_{i}}{\mu}\left(1+\frac{r}{\varkappa_{i}^{4} E J}\right) S\left(\varkappa_{i} d\right) a_{6}\right]=0
\end{aligned}
$$

Defining a set of values $k_{i}$ and $\varkappa$ we perform introducing constant $\varepsilon_{i^{*}}$ Using expressions (14) and (15), we determine the values $\omega_{i \varkappa}$ circular frequencies of natural transverse vibrations of a part of the beam $\alpha L$ on an elastic foundation and the values $\omega_{i k}$ for part of the $\beta L$ beam without base.

In order to determine the modes of natural vibrations, the values of the roots $k_{i}$ and $\varkappa$ substitute in the solution of the first equation (18), which determines the values of the relative ordinates of $i$-th form of natural vibrations.

The third stage.

Let us determine the efforts under the action of a dynamic load $F(t)=F \sin y t$, applied at an arbitrary point $d$ (Figure 3 ) for the same beam.

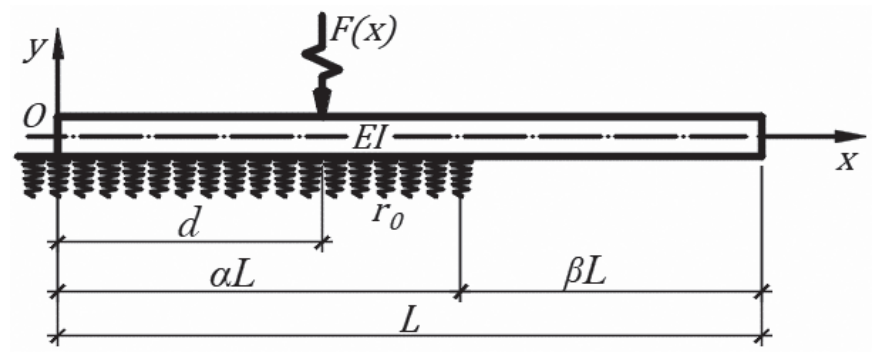

Figure 3. Beam with dynamic force $F(t)$

Let's return to the differential equation of forced vibrations of the beam (1). We assume that the disturbing force acts according to the law $q(x, t)=q(x)$ $\sin y t$. Assuming that forced vibrations also change according to a harmonic law:

$$
y(x, t)=\varphi(x) \sin (y t),
$$

we obtain an inhomogeneous differential equation of forced vibrations of a beam on an elastic foundation:

$$
\varphi^{I V}(x)+\varkappa^{4} \varphi(x)=q(x),
$$

where: $\varkappa^{4}=\frac{\mu \gamma^{2}-r}{E I}$.

For a beam without a base, the inhomogeneous differential equation of forced vibrations takes the form:

$$
\varphi^{I V}(x)+k^{4} \varphi(x)=q(x),
$$

where: $k^{4}=\frac{\mu \gamma^{2}}{E I}$.

We have obtain the general solutions of the inhomogeneous equations (22) and (24) as the sum of the general solutions of the homogeneous equation and the particular solution, which depends on the type of load. Further, using the method of initial parameters, we have obtain universal formulas for determining deflections, angles of rotation, moments and shear forces for an arbitrary section of the beam in the general case of the action of a disturbing load $q(x, t)$.

We use the values of the boundary conditions on the left and right edges of the beam (8).

For an arbitrary section of the beam in the first section $0 \leq x_{1} \leq \alpha L$, that located on an elastic foundation and under the action of a dynamic load $F(t)$, that applied at an arbitrary point $d$, displacements and forces are determined by the equations: 


$$
\left.\begin{array}{l}
y_{1 i}\left(x_{1}\right)=y_{10 i} S\left(\varkappa_{i} x_{1}\right)+\theta_{10 i} \frac{1}{\varkappa_{i}} T\left(\varkappa_{i} x_{1}\right)+\frac{F \sin \gamma t}{\varkappa_{i}{ }^{3} E I} V\left[\varkappa_{i}\left(x_{1}-d\right)\right] \\
\theta_{1 i}\left(x_{1}\right)=y_{10 i} \varkappa_{i} V\left(\varkappa_{i} x_{1}\right)+\theta_{10 i} S\left(\varkappa_{i} x_{1}\right)+\frac{F \sin \gamma t}{\varkappa_{i}{ }^{2} E I} U\left[\varkappa_{i}\left(x_{1}-d\right)\right] \\
M_{1 i}\left(x_{1}\right)=-E J y_{10 i} \varkappa_{i}^{2} U\left(\varkappa_{i} x_{1}\right)-E J \theta_{10 i} \varkappa_{i} V\left(\varkappa_{i} x_{1}\right)-\frac{F \sin \gamma t}{\varkappa_{i}} T\left[\varkappa_{i}\left(x_{1}-d\right)\right] \\
Q_{1 i}\left(x_{1}\right)=-E J y_{10 i} \varkappa_{i}^{3} T\left(\varkappa_{i} x_{1}\right)-E J \theta_{10 i} \varkappa_{i}^{2} U\left(\varkappa_{i} x_{1}\right)-F \sin \gamma t S\left[\varkappa_{i}\left(x_{1}-d\right)\right]
\end{array}\right\}
$$

In the second section of the beam without a base $0 \leq x_{2} \leq \beta L$ the displacements and forces for an arbitrary section are determined by (10). Using the conditions of conjugation of the sections and, expressing the displacements and forces of the second section through the initial parameters of the first section, we get:

$$
\begin{aligned}
& y_{2 i}\left(x_{2}\right)=y_{10 i}\left[S\left(\varkappa_{i} \alpha L\right) S\left(k_{i} x_{2}\right)+\frac{\varkappa_{i}}{k_{i}} V\left(\varkappa_{i} \alpha L\right) T\left(k_{i} x_{2}\right)+\frac{\varkappa_{i}^{2}}{k_{i}^{2}} U\left(\varkappa_{i} \alpha L\right) U\left(k_{i} x_{2}\right)+\frac{\varkappa_{i}^{3}}{k_{i}^{3}} T\left(\varkappa_{i} \alpha L\right) V\left(k_{i} x_{2}\right)\right]+ \\
& +\theta_{10 i}\left[\frac{1}{\varkappa_{i}} T\left(\varkappa_{i} \alpha L\right) S\left(k_{i} x_{2}\right)+\frac{1}{k_{i}} S\left(\varkappa_{i} \alpha L\right) T\left(k_{i} x_{2}\right)+\frac{\varkappa_{i}}{k_{i}^{2}} V\left(\varkappa_{i} \alpha L\right) U\left(k_{i} x_{2}\right)+\frac{\varkappa_{i}^{2}}{k_{i}^{3}} U\left(\varkappa_{i} \alpha L\right) V\left(k_{i} x_{2}\right)\right]+ \\
& +\frac{F \sin \gamma t}{E I}\left[\frac{V\left[\varkappa_{i}(\alpha L-d)\right]}{\varkappa_{i}{ }^{3}} S\left(k_{i} x_{2}\right)+\frac{U\left[\varkappa_{i}(\alpha L-d)\right]}{\varkappa_{i}{ }^{2} k_{i}} T\left(k_{i} x_{2}\right)+\frac{T\left[\varkappa_{i}(\alpha L-d)\right]}{\varkappa_{i} k_{i}^{2}} U\left(k_{i} x_{2}\right)+\frac{S\left[\varkappa_{i}(\alpha L-d)\right]}{k_{i}^{3}} V\left(k_{i} x_{2}\right)\right] \\
& \theta_{2 i}\left(x_{2}\right)=y_{10 i}\left[k_{i} S\left(\varkappa_{i} \alpha L\right) V\left(k_{i} x_{2}\right)+\varkappa_{i} V\left(\varkappa_{i} \alpha L\right) S\left(k_{i} x_{2}\right)+\frac{\varkappa_{i}^{2}}{k_{i}} U\left(\varkappa_{i} \alpha L\right) T\left(k_{i} x_{2}\right)+\frac{\varkappa_{i}^{3}}{k_{i}^{2}} T\left(\varkappa_{i} \alpha L\right) U\left(k_{i} x_{2}\right)\right]+ \\
& +\theta_{10 i}\left[\frac{k_{i}}{\varkappa_{i}} T\left(\varkappa_{i} \alpha L\right) V\left(k_{i} x_{2}\right)+S\left(\varkappa_{i} \alpha L\right) S\left(k_{i} x_{2}\right)+\frac{\varkappa_{i}}{k_{i}} V\left(\varkappa_{i} \alpha L\right) T\left(k_{i} x_{2}\right)+\frac{\varkappa_{i}^{2}}{k_{i}^{2}} U\left(\varkappa_{i} \alpha L\right) U\left(k_{i} x_{2}\right)\right]+ \\
& +\frac{F \sin \gamma t}{E I}\left[\frac{V\left[\varkappa_{i}(\alpha L-d)\right]}{\varkappa_{i}{ }^{3}} k_{i} V\left(k_{i} x_{2}\right)+\frac{U\left[\varkappa_{i}(\alpha L-d)\right]}{\varkappa_{i}{ }^{2}} S\left(k_{i} x_{2}\right)+\frac{T\left[\varkappa_{i}(\alpha L-d)\right]}{\varkappa_{i} k_{i}} T\left(k_{i} x_{2}\right)+\frac{S\left[\varkappa_{i}(\alpha L-d)\right]}{k_{i}^{2}} U\left(k_{i} x_{2}\right)\right] \\
& M_{2 i}\left(x_{2}\right)=-E J y_{10 i}\left[k_{i}^{2} S\left(\varkappa_{i} \alpha L\right) U\left(k_{i} x_{2}\right)+\varkappa_{i} k_{i} V\left(\varkappa_{i} \alpha L\right) V\left(k_{i} x_{2}\right)+\varkappa_{i}^{2} U\left(\varkappa_{i} \alpha L\right) S\left(k_{i} x_{2}\right)+\frac{\varkappa_{i}^{3}}{k_{i}} T\left(\varkappa_{i} \alpha L\right) T\left(k_{i} x_{2}\right)\right]- \\
& -E J \theta_{10 i}\left[\frac{k_{i}^{2}}{\varkappa_{i}} T\left(\varkappa_{i} \alpha L\right) U\left(k_{i} x_{2}\right)+k_{i} S\left(\varkappa_{i} \alpha L\right) V\left(k_{i} x_{2}\right)+\varkappa_{i} V\left(\varkappa_{i} \alpha L\right) S\left(k_{i} x_{2}\right)+\frac{\varkappa_{i}^{2}}{k_{i}} U\left(\varkappa_{i} \alpha L\right) T\left(k_{i} x_{2}\right)\right]- \\
& -\frac{F \sin \gamma t}{\varkappa_{i}{ }^{3}}\left[k_{i}^{2} U\left(k_{i} x_{2}\right) V\left[\varkappa_{i}(\alpha L-d)\right]+\varkappa_{i} k_{i} V\left(k_{i} x_{2}\right) U\left[\varkappa_{i}(\alpha L-d)\right]+\varkappa_{i}^{2} T\left[\varkappa_{i}(\alpha L-d)\right] S\left(k_{i} x_{2}\right)++\frac{\varkappa_{i}^{3}}{k_{i}} S\left[\varkappa_{i}(\alpha L-d)\right] T\left(k_{i} x_{2}\right)\right] \\
& Q_{2 i}\left(x_{2}\right)=-E J y_{10 i}\left[k_{i}^{3} T\left(k_{i} x_{2}\right) S\left(\varkappa_{i} \alpha L\right)+\varkappa_{i} k_{i}^{2} U\left(k_{i} x_{2}\right) V\left(\varkappa_{i} \alpha L\right)+\varkappa_{i}^{2} k_{i} \mathrm{~V}\left(k_{i} x_{2}\right) U\left(\varkappa_{i} \alpha L\right)+\varkappa_{i}^{3} \mathrm{~S}\left(k_{i} x_{2}\right) T\left(\varkappa_{i} \alpha L\right)\right]- \\
& -E J \theta_{10 i}\left[\frac{k_{i}^{3}}{\varkappa_{i}} T\left(\varkappa_{i} \alpha L\right) T\left(k_{i} x_{2}\right)+k_{i}^{2} U\left(k_{i} x_{2}\right) S\left(\varkappa_{i} \alpha L\right)+\varkappa_{i} k_{i} V\left(\varkappa_{i} \alpha L\right) \mathrm{V}\left(k_{i} x_{2}\right)+\varkappa_{i}^{2} U\left(\varkappa_{i} \alpha L\right) \mathrm{S}\left(k_{i} x_{2}\right)\right]- \\
& \left.-\frac{F \sin \gamma t}{\varkappa_{i}{ }^{3}}\left[k_{i}^{3} T\left(k_{i} x_{2}\right) V\left[\varkappa_{i}(\alpha L-d)\right]+\varkappa_{i} k_{i}^{2} U\left[\varkappa_{i}(\alpha L-d)\right] U\left(k_{i} x_{2}\right)+\varkappa_{i}^{2} k_{i} \mathrm{~V}\left(k_{i} x_{2}\right) T\left[\varkappa_{i}(\alpha L-d)\right]++\mathcal{\varkappa}_{i}^{3} \mathrm{~S}\left(k_{i} x_{2}\right) S\left[\mathcal{\varkappa}_{i}(\alpha L-d)\right]\right]\right)
\end{aligned}
$$

We denote:

$$
\begin{aligned}
a_{1}= & {\left[k_{i}^{2} S\left(\varkappa_{i} \alpha L\right) U\left(k_{i} \beta L\right)+\varkappa_{i} k_{i} V\left(\varkappa_{i} \alpha L\right) V\left(k_{i} \beta L\right)+\varkappa_{i}^{2} U\left(\varkappa_{i} \alpha L\right) S\left(k_{i} \beta L\right)+\frac{\varkappa_{i}^{3}}{k_{i}} T\left(\varkappa_{i} \alpha L\right) T\left(k_{i} \beta L\right)\right] ; } \\
a_{2}= & {\left[\frac{k_{i}^{2}}{\varkappa_{i}} T\left(\varkappa_{i} \alpha L\right) U\left(k_{i} \beta L\right)+k_{i} S\left(\varkappa_{i} \alpha L\right) V\left(k_{i} \beta L\right)+\varkappa_{i} V\left(\varkappa_{i} \alpha L\right) S\left(k_{i} \beta L\right)+\frac{\varkappa_{i}^{2}}{k_{i}} U\left(\varkappa_{i} \alpha L\right) T\left(k_{i} \beta L\right)\right] ; } \\
a_{3}= & {\left[k_{i}^{2} U\left(k_{i} \beta L\right) V\left[\varkappa_{i}(\alpha L-d)\right]+\varkappa_{i} k_{i} V\left(k_{i} \beta L\right) U\left[\varkappa_{i}(\alpha L-d)\right]+\varkappa_{i}^{2} T\left[\varkappa_{i}(\alpha L-d)\right] S\left(k_{i} \beta L\right)+\right.} \\
& \left.+\frac{\varkappa_{i}^{3}}{k_{i}} S\left[\varkappa_{i}(\alpha L-d)\right] T\left(k_{i} \beta L\right)\right] ; \\
a_{4}= & {\left[k_{i}^{3} T\left(k_{i} \beta L\right) S\left(\varkappa_{i} \alpha L\right)+\varkappa_{i} k_{i}^{2} U\left(k_{i} \beta L\right) V\left(\varkappa_{i} \alpha L\right)+\varkappa_{i}^{2} k_{i} \mathrm{~V}\left(k_{i} \beta L\right) U\left(\varkappa_{i} \alpha L\right)+\varkappa_{i}^{3} \mathrm{~S}\left(k_{i} \beta L\right) T\left(\varkappa_{i} \alpha L\right)\right] ; } \\
a_{5}= & {\left[\frac{k_{i}^{3}}{\varkappa_{i}} T\left(\varkappa_{i} \alpha L\right) T\left(k_{i} \beta L\right)+k_{i}^{2} U\left(k_{i} \beta L\right) S\left(\varkappa_{i} \alpha L\right)+\varkappa_{i} k_{i} V\left(\varkappa_{i} \alpha L\right) \mathrm{V}\left(k_{i} \beta L\right)+\varkappa_{i}^{2} U\left(\varkappa_{i} \alpha L\right) \mathrm{S}\left(k_{i} \beta L\right)\right] ; } \\
a_{6}= & {\left[k_{i}^{3} T\left(k_{i} \beta L\right) V\left[\varkappa_{i}(\alpha L-d)\right]+\varkappa_{i} k_{i}^{2} U\left[\varkappa_{i}(\alpha L-d)\right] U\left(k_{i} \beta L\right)+\varkappa_{i}^{2} k_{i} \mathrm{~V}\left(k_{i} \beta L\right) T\left[\varkappa_{i}(\alpha L-d)\right]+\right.} \\
& \left.+\varkappa_{i}^{3} \mathrm{~S}\left(k_{i} \beta L\right) S\left[\varkappa_{i}(\alpha L-d)\right]\right] .
\end{aligned}
$$


Using the boundary conditions on the right edge (8) for $x_{2}=\beta L$, we obtain a system of equations for determining $y_{10 \mathrm{i}}$ and $\theta_{10 \mathrm{i}}$ :

$$
\left\{\begin{array}{l}
a_{1} y_{10 i}+a_{2} \theta_{10 i}=\frac{-F \sin \gamma t}{E J \varkappa_{i}^{3}} a_{3} \\
a_{4} y_{10 i}+a_{5} \theta_{10 i}=\frac{-F \sin \gamma t}{E J \varkappa_{i}^{3}} a_{6}
\end{array}\right.
$$

Using (26) and (28), at a given frequency of forced oscillations $\gamma$, we determine $\varkappa$ and $k$ :

$$
\begin{aligned}
& x=\sqrt[4]{\frac{\mu \gamma^{2}-r}{E J}}, \\
& k=\sqrt[4]{\frac{\mu \gamma^{2}}{E J}} .
\end{aligned}
$$

Applying equations (26) and (27) taking into account certain values of the roots $\varkappa$ and $k$, that corresponds to given frequency $\gamma$ of forced vibrations, and values of $F(t)$, we have determine forces in the beam under forced vibrations.

\section{RESULTS AND ANALYSIS}

Initial for calculations: beam width $b=1.25 \mathrm{~m}$, height $h=1.5 \mathrm{~m}$, length $L=12.0 \mathrm{~m}$, elasticity modulus of material $E=2,1 \times 10^{6} \mathrm{t} / \mathrm{m}^{2}$, modulus of subgrade reaction $r_{0}=5000 \mathrm{t} / \mathrm{m}^{3}$, force $F=10.0 \mathrm{t}$, mass $m_{1}=$ $F / g=1.0194 \mathrm{t}$.

At the first and second stages, according to the results of calculations of the beam with $\alpha L=\beta L$, the values of the roots $\varkappa_{i}$ and $k_{i}$ of the equations (13) and (20) are adopted such that $\varepsilon_{i}$ from $k_{i}=\varepsilon_{i} \varkappa_{i}$ equal $0.5 ; 1.0$ and 2.0. Also, the value $\varepsilon_{i}$ is taken from the condition of equality of natural frequencies of transverse vibrations $\omega_{i x}=\omega_{i k}$ of two parts of beam. Root values $\varkappa_{i}$ and $k_{i}$, indicated in column 7 of tables 1 and 2 for a beam on a full base are defined in [15], in column 8 for a beam without a base - in [14]. The calculation results are presented in table 1 .

At the first stage, the first three modes of beam vibrations were constructed with $\alpha L=\beta L$ without added mass $m_{1}$ (Figures 4, 5 and 6) corresponding to natural frequencies for $\varepsilon$.

Further, at the second stage, according to the results of calculations of a beam with an added mass $m_{1}$ located in a quarter of the beam $d=L / 4$ at $\alpha L=$

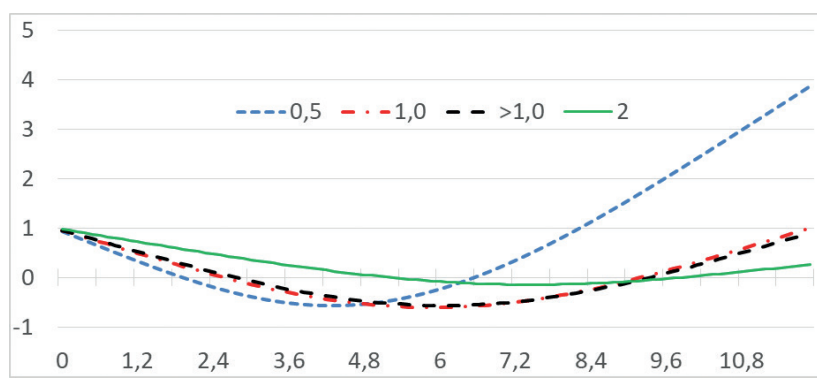

Figure 4.1st mode of vibration with $\alpha L=\beta L$ without mass $m_{1}$

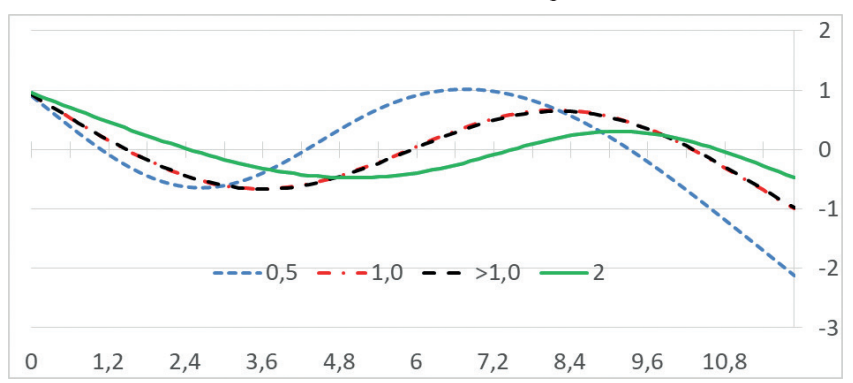

Figure 5.The 2nd mode of vibration at $\alpha L=\beta L$ without mass $m_{1}$

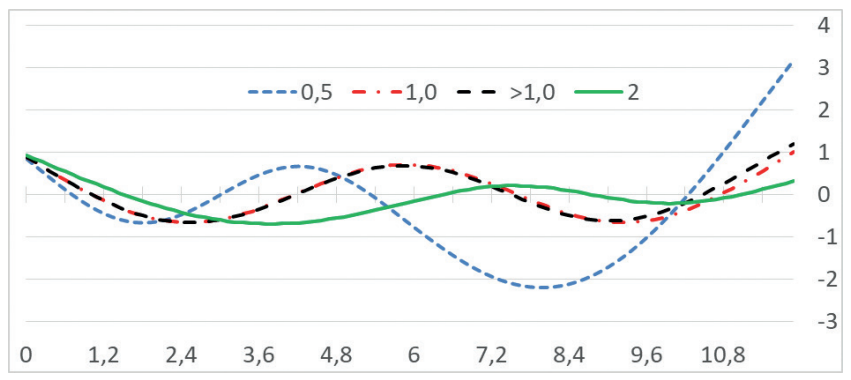

Figure 6.The 3rd mode of vibration at $\alpha L=\beta L$ without mass $m_{1}$

\begin{tabular}{|c|c|c|c|c|c|c|c|}
\hline & & \multicolumn{4}{|c|}{$\varepsilon_{i}$} & \multirow[b]{2}{*}{ 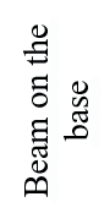 } & \multirow[b]{2}{*}{ 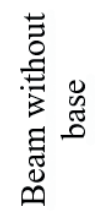 } \\
\hline & & 0.5 & 1.0 & 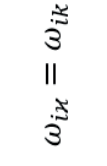 & 2.0 & & \\
\hline 1 & 2 & 3 & 4 & 5 & 6 & 7 & 8 \\
\hline \multirow{4}{*}{$\begin{array}{l}\stackrel{\mathscr{Z}}{0} \\
\text { ఏ }\end{array}$} & $\varkappa_{1}$ & 0.5264 & \multirow{2}{*}{0.3942} & 0.3762 & 0.2632 & \begin{tabular}{|l|}
0.3942 \\
\end{tabular} & - \\
\hline & $k_{1}$ & 0.2632 & & 0.4108 & 0.5264 & - & 0.3942 \\
\hline & $\omega_{1}^{x}$ & 254.7 & 161.7 & \multirow{2}{*}{228.9} & 156.2 & 161.7 & - \\
\hline & $\omega_{1}{ }^{k}$ & 59.4 & 133.3 & & 375.8 & - & 133.3 \\
\hline \multirow{4}{*}{$\begin{array}{l}\stackrel{0}{0} \\
\text { d } \\
\text { N }\end{array}$} & $\varkappa_{1}$ & 0.8739 & \multirow{2}{*}{0.6544} & 0.6507 & 0.4369 & 0.6544 & - \\
\hline & $k_{2}$ & 0.4369 & & 0.6582 & 0.8738 & - & 0.6544 \\
\hline & $\omega_{2}{ }^{x}$ & 661.4 & 378.5 & & 287.4 & 378.5 & - \\
\hline & $\omega_{2}{ }^{k}$ & 163.7 & 367.3 & $58 \% .6$ & 1035.5 & - & 367.3 \\
\hline \multirow{4}{*}{ 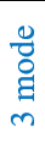 } & $x_{1}$ & 1.2566 & \multirow{2}{*}{0.9163} & 0.9149 & 0.6283 & 0.9163 & - \\
\hline & $k_{3}$ & 0.6283 & & 0.9177 & 1.2566 & - & 0.9163 \\
\hline & $\omega 3^{x}$ & 1357.5 & 726.0 & \multirow{2}{*}{1142.2} & 549.7 & 726.0 & - \\
\hline & $\omega_{3}{ }^{k}$ & 338.6 & 720.2 & & 2141.6 & & 720.2 \\
\hline
\end{tabular}

Table 1. Roots and natural angular frequencies ( $\mathrm{rad}$ / sec) of transverse vibrations for $\alpha L=\beta$. 
$\beta L$, we obtain the values of the roots $\varkappa$ and $k_{i}$. The calculation results are presented in Table 2.

The first three modes of vibrations of the beam are constructed for $\alpha L=\beta L$ with the added mass m1 located at the point $d=L / 4$. Vibration modes corresponding to natural frequencies for $\varepsilon$ are presented in Figures 7, 8 and 9.

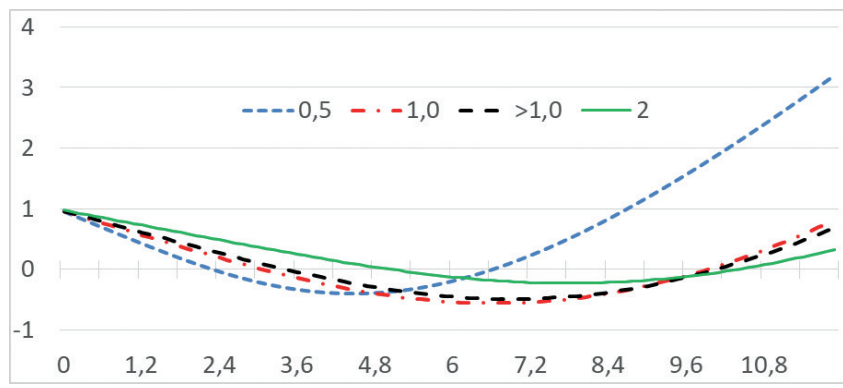

Figure 7. 1st mode of vibration at $\alpha L=\beta L$ with mass $m_{1}$ at point $d=L / 4$

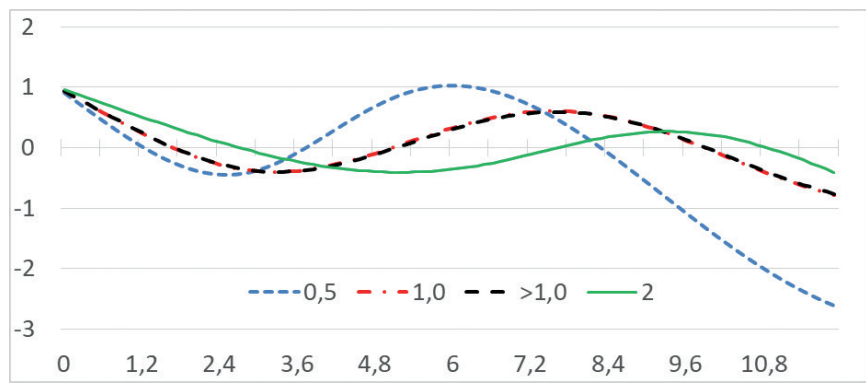

Figure 8. 2nd mode of vibration at $\alpha L=\beta L$ with mass $m_{1}$ at point $d=L / 4$

Table 2. Roots and natural angular frequencies ( $\mathrm{rad}$ / s) of transverse vibrations at $\alpha L=\beta$ with mass $\mathrm{m}_{1}$ at point $\mathrm{d}=\mathrm{L} / 4$

\begin{tabular}{|c|c|c|c|c|c|c|c|}
\hline & & \multicolumn{4}{|c|}{$\varepsilon_{i}$} & \multirow[b]{2}{*}{ 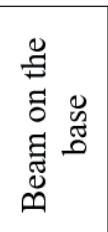 } & \multirow[b]{2}{*}{ 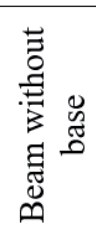 } \\
\hline & & 0.5 & 1.0 & 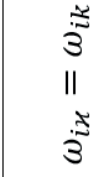 & 2.0 & & \\
\hline & 2 & & & 5 & & & 8 \\
\hline \multirow{4}{*}{$\begin{array}{l}\text { ष्. } \\
\text { : }\end{array}$} & $\varkappa_{1}$ & 0.4927 & \multirow{2}{*}{0.3364} & 0.3034 & 0.2042 & 0.3925 & \\
\hline & $k_{1}$ & 0.2463 & & \begin{tabular}{|l|}
0.3607 \\
\end{tabular} & 0.4085 & & 0.3942 \\
\hline & $\omega_{1}{ }^{x}$ & 352.1 & 197.8 & \multirow{2}{*}{176.5} & 137.0 & 160.7 & - \\
\hline & $\omega_{1}{ }^{k}$ & 82.3 & 153.8 & & 226.3 & - & 133.3 \\
\hline \multirow{4}{*}{$\begin{array}{l}\mathbb{z} \\
\text { ב } \\
\text { N }\end{array}$} & $x_{1}$ & 0.8466 & \multirow{2}{*}{0.6521} & 0.6477 & 0.4168 & 0.6358 & - \\
\hline & $k_{2}$ & 0,4233 & & \begin{tabular}{|l|}
0.6553 \\
\end{tabular} & 0.8336 & - & 0.6544 \\
\hline & $\omega_{2}{ }^{x}$ & 980.0 & 590.1 & \multirow{2}{*}{582.5} & 266.6 & 358.6 & - \\
\hline & $\omega_{2}{ }^{k}$ & 243 & 576.7 & & 942.4 & 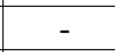 & 367.3 \\
\hline \multirow{4}{*}{ 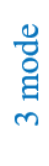 } & $\varkappa_{1}$ & 1.3749 & \multirow{2}{*}{0.} & 0.88 & 0.62 & 0.8936 & - \\
\hline & $k_{3}$ & 0.6874 & & 0.8831 & 1.24 & - & 0.9163 \\
\hline & $\omega_{3^{x}}$ & 2565.7 & 1064.3 & \multirow{2}{*}{1057.7} & 536.1 & 691.0 & - \\
\hline & $\omega_{3}{ }^{k}$ & 640.8 & 1057.0 & & 2085.3 & & 720.2 \\
\hline
\end{tabular}

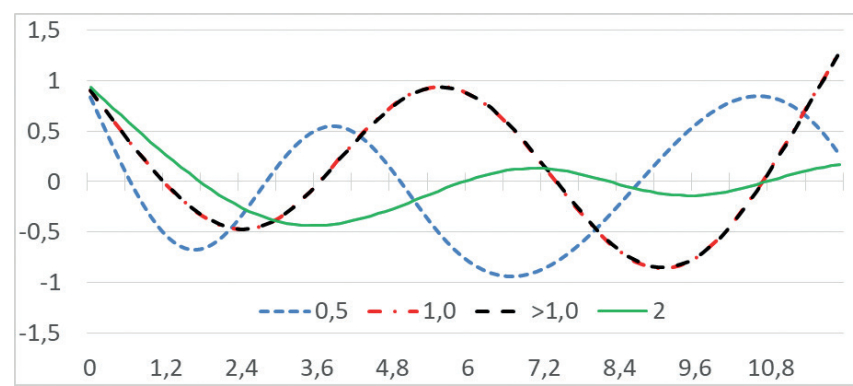

Figure 9. The 3rd mode of vibration at $\alpha L=\beta L$ with mass $m_{1}$ at point $d=L / 4$

At the third stage, an example with the same beam under the action of a disturbing force $F=10.0 \mathrm{t}$, applied in the points $d=L / 2$ and $d=L / 4$ is considered. Forced vibration frequencies are $\gamma_{1}=220 \mathrm{rad} / \mathrm{s}$ and $\gamma_{2}=400 \mathrm{rad} / \mathrm{s}$. The displacements and forces in the beams are determined at various values $\alpha L$. The figures 10-13 show the bending moment plots.

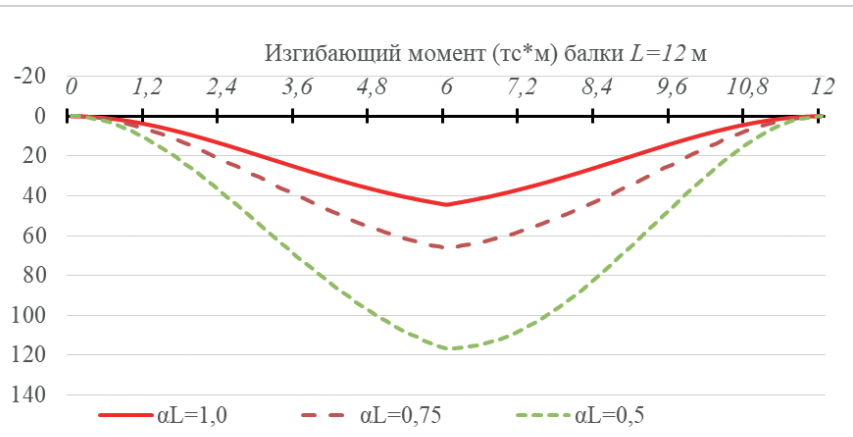

Figure 10. Diagrams of bending moments under the action of the force $F(t)$ at the point $d=L / 2$ at $\gamma_{1}=220 \mathrm{rad} / \mathrm{s}$

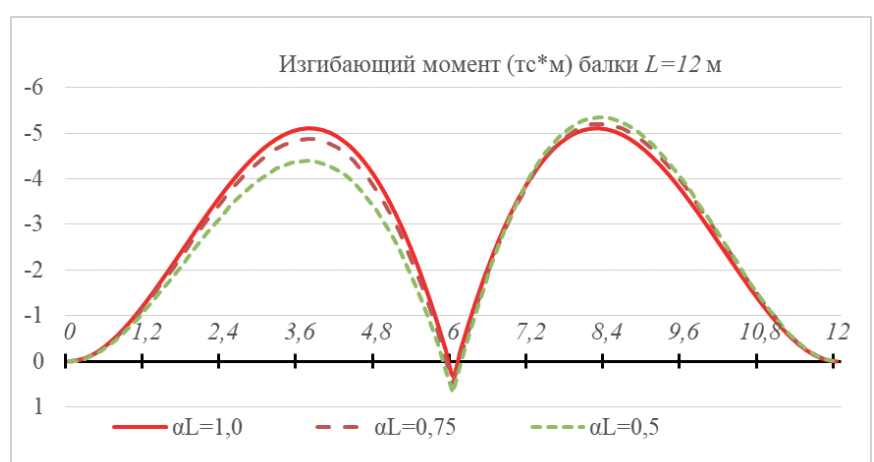

Figure 11. Diagrams of bending moments under the action of the force $F(t)$ at the point $d=L / 2$ at $\gamma_{2}=400 \mathrm{rad} / \mathrm{s}$ 


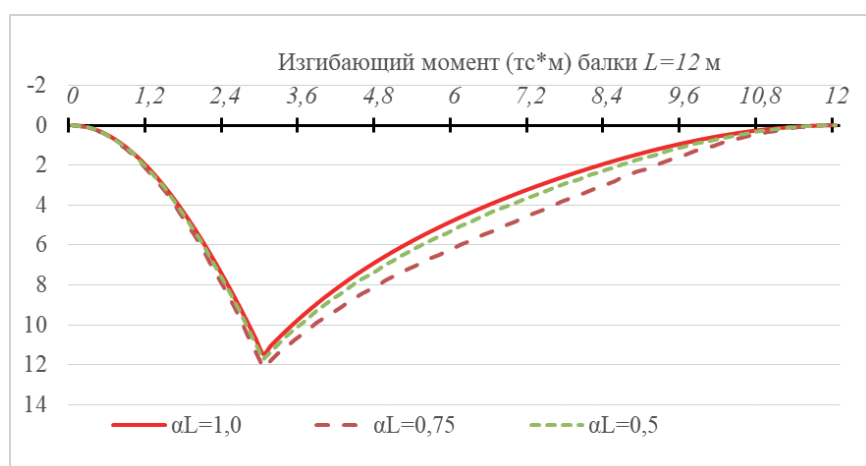

Figure 12. Diagrams of bending moments under the action of the force $F(t)$ at the point $d=L / 4$ at $\gamma_{1}=220 \mathrm{rad} / \mathrm{s}$

Analysis of the calculation results of the third stage allows us to draw the following conclusions.

The action of a disturbing force in the middle of the beam $(d=L / 2)$ with a frequency of forced vibrations $\gamma=220 \mathrm{rad} / \mathrm{s}$, close to the frequency of natural vibrations (for $\omega_{i \varkappa}=\omega_{i k}$ ) for the first mode of vibration, leads to an increase in displacements and efforts in sections of the beam more than three times when excluding part of the base from the work. Under similar conditions, the action of a disturbing force with a forced vibration frequency $\gamma=400 \mathrm{rad}$ / $\mathrm{s}$, close to the natural vibration frequency for the second form, does not lead to a significant change in the forces in the beam sections when part of the base is excluded from operation.

The action of a disturbing force in a quarter of the beam $(\mathrm{d}=\mathrm{L} / 4)$ with a frequency of forced vibrations $\gamma=220 \mathrm{rad} / \mathrm{s}$ and $\gamma=400 \mathrm{rad} / \mathrm{s}$, when part of the base is excluded from the work, does not lead to a significant change in the forces in the beam sections.

\section{CONCLUSIONS}

1) under different conditions of support of the "beam-base" system, different frequencies of natural vibrations based on the results of calculating the roots of the secular equation can correspond to different parts of one beam. The values of the roots that determine the main modes of vibration of the beam as a whole are the values of the roots for a part of the beam on the base, while the natural frequency of the transverse vibrations of the part of the beam on the base is greater than or equal to the natural frequency of vibration of the part of the beam without the base;

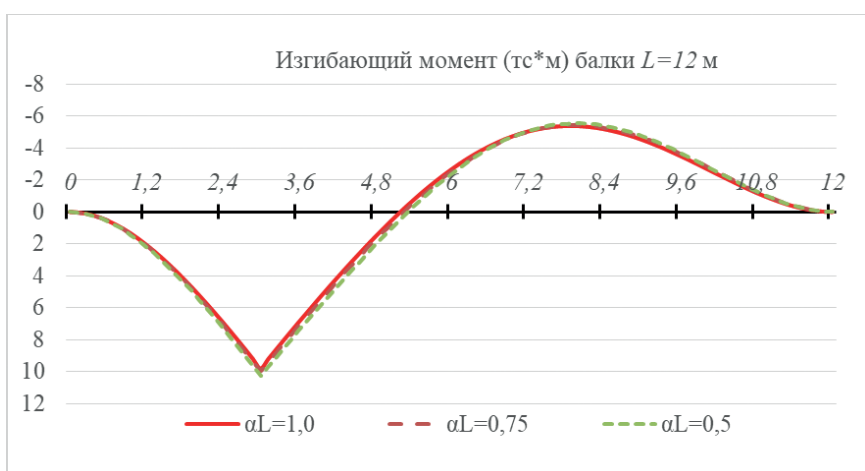

Figure 13. Diagrams of bending moments under the action of the force $F(t)$ at the point $d=L / 4$ at $\gamma_{2}=400 \mathrm{rad} / \mathrm{s}$

2) under different conditions of support of the "beambase" system, the values of the natural frequencies of transverse vibrations can be equal for different modes of vibration of two different parts of the beam. In this case, the action of a disturbing force with a frequency of forced vibrations equal to the frequency of natural vibrations leads to the formation of resonance for each of the two different modes of vibration of each part of the beam;

3) with the application of an additional mass $\mathrm{ml}$ at the beam point, the vibration frequencies change its values. If the mass of the system increases, then the vibration frequencies of the system decrease and vice versa that corresponds to a similar conclusion for a beam on a full base [15];

4) when performing a dynamic calculation, it is necessary to consider all possible options for the application of masses, taking into account the points of their location in combinations with options for changing the conditions for supporting the beam on an elastic foundation. The number of determined frequencies and modes of natural vibrations for beams on an elastic foundation should not be less than two;

These conditions must be taken into account when analyzing the dynamic behavior of a structure under the action of variable loads in the event of a change in the conditions of bearing on an elastic foundation.

\section{REFERENCES}

1.Federal law of 30.12.2010 No 384-FZ «Tekhnicheskiy reglament o bezopasnosti zdaniy i sooruzheniy» [Technical Regulations on the Safety of 
Buildings and Structures] (entered 1 Jule 2010). - Novosibirsk: Sib. Univ. Izd-vo, 2010. - 31 p.

2. Russian Standard GOST 27751-2014 «Nadezhnost' stroitel'nykh konstruktsiy i osnovaniy. Osnovnyye polozheniya» [Reliability of building structures and foundations. Basic provisions]. Mocow, Standartinform, 2015.

3. Russian Building Code SP 385.1325800.2018 «Zashchita zdaniy i sooruzheniy ot progressiruyushchego obrusheniya. Pravila proyektirovaniya. Osnovnyye polozheniya» [Protection of buildings and structures from progressive collapse. Design rules. Basic provisions]. Minstroy Rossii, 2018.

4. Travush V.I., Kolchunov V.I., Leont'yev Ye.V. Zashchita zdaniy i sooruzheniy of progressiruyushchego obrusheniya $\mathrm{v}$ ramkakh zakonodatel'nykh i normativnykh trebovaniy [Protection of buildings and structures from progressive collapse within the framework of legislative and regulatory requirements] // Promyshlennoye i grazhdanskoye stroitel'stvo. 2019. No 2. P. 46-54.

5. Travush V.I., Fedorova N.V. Zhivuchest' konstruktivnykh sistem sooruzheniy pri osobykh vozdeystviyakh [Survivability of structural systems of structures under special influences] // Magazine of Civil Engineering. 2018. No 5(81). P. 73-80. doi:10.18720/MCE. 81.8

6. Travush V.I., Shapiro G.I., Kolchunov V.I., Leont'yev Ye.V., Fedorova N.V. Proyektirovaniye zashchity krupnopanel'nykh zdaniy ot progressiruyushchego obrusheniya [Designing the protection of large-panel buildings from progressive collapse] // Zhilishchnoye stroitel'stvo. 2019. No 3. P. 40-46. DOI: 10.31659/0044-4472-2019-3-40-46

7. Travush V.I., etc. The response of the system «beam - Foundation» on sudden changes of boundary conditions // IOP Conf. Ser. Mater. Sci. Eng. 2018. № 1(456). 012130.

8. Travush V.I., Gordon V.A., Kolchunov V.I., Leontiev Y.V. Dynamic effects in the beam on an elastic foundation caused by the sudden transformation of supporting conditions // International Journal for Computational Civil and Structural Engineering. 2018. 14 (4). Pp. 27-41. DOI: $10.22337 / 2587-9618-2018-14-4-27-47$
9. Travush V.I., Gordon V.A., Kolchunov V.I., Leontiev E.V. Dynamic deformation of a beam at sudden structural transformation of foundation. Magazine of Civil Engineering. 2019. 91(7). Pp. 129-144. DOI: 10.18720/MCE.91.12

10. Aleksandrov, A.V. Soprotivleniye materialov [Resistance of materials] / A.V.Aleksandrov, V.D.Potapov, B.P.Derzhavin. - 8-th edition, remastered. - M.: Student, 2012. - 560 p.

11. Krylov, A.N. O raschete balok, lezhashchikh na uprugom osnovanii [bout the calculation of beams lying on an elastic foundation] / A.N.Krylov. - 3-rd edition. - Leningrad: Publ. AN SSSR, 1930. - 154 p.

12. Gorbunov-Posadov, M.I., Raschet konstruktsiy na uprugom osnovanii [Calculation of structures on an elastic foundation] / M.I.GorbunovPosadov, T.A. Malikova. - M., Stroyizdat, 1973. $-627 \mathrm{p}$

13. Korenev, B.G. Dinamicheskiy raschet zdaniy i sooruzheniy [Dynamic calculation of buildings and structures] / M.F.Barshteyn, V.A.Il'ichev, B.G.Korenev et al. - 2-nd edition, remastered and added. - M.:Stroyizdat, 1984. -303 p.

14. Babakov, I.M. Teoriya kolebaniy [Theory of oscillations] / Babakov I.M. - M:. Nauka, 1965 $-560 \mathrm{p}$.

15. Leont'yev Ye.V. Poperechnyye kolebaniya balki na uprugom osnovanii pri deystvii dinamicheskoy nagruzki [Transverse vibrations of a beam on an elastic foundation under a dynamic load] // Stroitel'stvo i rekonstruktsiya. - 2020. № 3 (89). P. 31-44.

\section{СПИСОК ЛИТЕРАТУРЫ}

1. Федеральный закон от 30.12.2010 № 384-Ф3 «Технический регламент о безопасности зданий и сооружений» (вступил в силу с 1 июля 2010 года). - Новосибирск: Сиб. Унив. Изд-во, 2010. $-31 \mathrm{c}$.

2. ГОСТ 27751-2014 «Надежность строительных конструкций и оснований. Основные положения». Москва, Стандартинформ, 2015.

3. СП 385.1325800.2018 «Защита зданий и сооружений от прогрессирующего обрушения. Правила проектирования. Основные положения» / Минстрой России, 2018. 
4. Травуш В.И., Колчунов В.И., Леонтьев Е.В. Защита зданий и сооружений от прогрессирующего обрушения в рамках законодательных и нормативных требований//Промышленное и гражданское строительство. 2019. № 2. С. $46-54$.

5. Травуш В.И., Федорова Н.В. Живучесть конструктивных систем сооружений при особых воздействиях // Инженерно-строительный журнал. 2018. № 5(81). С. 73-80. doi:10.18720/ MCE.81.8

6. Травуш В.И., Шапиро Г.И., Колчунов В.И., Леонтьев Е.В., Федорова Н.В. Проектирование защиты крупнопанельных зданий от прогрессирующего обрушения//Жилищное строительство. 2019. № 3. С. 40-46. DOI: 10.31659/0044-4472-2019-3-40-46

7. Travush V.I., etc. The response of the system «beam - Foundation» on sudden changes of boundary conditions // IOP Conf. Ser. Mater. Sci. Eng. 2018. № 1(456). 012130.

8. Travush V.I., Gordon V.A., Kolchunov V.I., Leontiev Y.V. Dynamic effects in the beam on an elastic foundation caused by the sudden transformation of supporting conditions // International Journal for Computational Civil and Structural Engineering. 2018.14 (4). Pp. 27-41. DOI: $10.22337 / 2587-9618-2018-14-4-27-47$

9. Travush V.I., Gordon V.A., Kolchunov V.I., Leontiev E.V. Dynamic deformation of a beam at sudden structural transformation of foundation. Magazine of Civil Engineering. 2019. 91(7). Pp. 129-144. DOI: 10.18720/MCE.91.12

10. Александров, А.В. Сопротивление материалов / А.В.Александров, В.Д.Потапов, Б.П.Державин. - 8-е изд., испр. - М.: Студент, 2012. - 560 с: ил.

11. Крылов, А.Н. О расчете балок, лежащих на упругом основании / А.Н.Крылов. - Изд. 3-е. - Ленинград: Изд-во АН СССР, 1930. - 154 с. - (Справочно-техническая литература). - [На обл.: 1931 г.]

12. Горбунов-Посадов, М.И., Расчет конструкций на упругом основании. / М.И.ГорбуновПосадов, Т.А. Маликова. - М., Стройиздат, 1973. $-627 \mathrm{c}$.

13. Коренев, Б.Г. Динамический расчет зданий и сооружений / М.Ф.Барштейн, В.А.Ильичев, Б.Г.Коренев и др. - 2-е изд., перераб. и доп. - М.:Стройиздат, 1984. - 303 с., ил. - (Справочник проектировщика).

14. Бабаков, И.М. Теория колебаний / Бабаков И.М. - М:. Наука, 1965 - 560 с., с илл.

15. Леонтьев Е.В. Поперечные колебания балки на упругом основании при действии динамической нагрузки. // Строительство и реконструкция. - Орел: Изд-во ФГБОУ ВО «ОГУ имени И.С. Тургенева». - 2020. № 3 (89). С. $31-44$.

Yevgeny $V$. Leontiev, Deputy Chief of Management of Construction Design, Chief of the Structural Reliability and Safety of Objects Department; Federal Autonomous Institution "Main Department of State Expertise"; 6 Furkasovsky pereulok, Moscow, 101000, Russia; phone +7 (495) 625-95-95, 540-70-96; E-mail: e.leontyev@gge.ru.

Леонтьев Евгений Владимирович, заместитель начальника Управления строительных решений - начальник отдела конструктивной надежности и безопасности объектов; ФАУ «Главгосэкспертиза России»; 101000, Россия, Москва, Фуркасовский пер., д. 6; тел. +7 (495) 625-95-95, E-mail: e.leontyev@gge.ru. 